\title{
"Surgical management of complex multiloculated hydrocephalus in infants and children." Child's Nervous System, February 2015, volume 31, issue 2, pp. 243-249
}

\author{
Dimitrios Paraskevopoulos
}

Received: 21 February 2015 / Accepted: 25 February 2015 /Published online: 21 March 2015

(C) Springer-Verlag Berlin Heidelberg 2015

Dear Editor,

We read with great interest the recent article "Surgical management of complex multiloculated hydrocephalus in infants and children" by Akbari et al. [1], concerning the open and endoscopic fenestration of complex cysts in multiloculated hydrocephalus.

The authors conducted a retrospective study by querying their operative database and identifying 25 subjects, 12 of which initially underwent open fenestration and 13 underwent endoscopic fenestration. This retrospective cohort study concluded that endoscopic fenestration may represent the preferred initial treatment for many of these cases, primarily due to lower surgical morbidity, and suggested that open fenestration may be best reserved for more severe cases or those that have failed prior endoscopic surgery [1].

Surgical fenestration of complex cysts and septations in multiloculated hydrocephalus, either by craniotomy or endoscopic means, has been used with the intent to simplify shunt systems and reduce the number of shunt catheters or subsequent repeated procedures such as shunt revisions $[2,3]$. Treatment of multiloculated hydrocephalus may prove to be quite challenging. The options described by the authors are the main most useful treatment alternatives, in order to minimize the number of repeat fenestrations and maximize the latency to subsequent shunt revisions.

A useful adjunct to the armamentarium of the neurosurgeon in complex cases is the combination of image guidance,

D. Paraskevopoulos $(\bowtie)$

St. Bartholomew's and The Royal London Hospital, London, UK e-mail: dimitris.parask@gmail.com intraoperative imaging and endoscopy. The rationale is that in cases where multiple fenestrations are required and no anatomical landmarks are visible to navigate clinically, the preoperative dataset accuracy of image guidance drops significantly after the first fenestration, due to brain shift and a change in the intraoperative situs, as CSF fluid dynamics change. The combination of these techniques has been described by several groups with various intraoperative MRI systems [4-6]. Obviously, the equipment can be quite expensive and is not available in every setup. However, it can prove to be a feasible and effective technique, which may complement standard endoscopic fenestrations in complex cases and should therefore be considered.

\section{References}

1. Akbari SH, Holekamp TF, Murphy TM, Mercer D, Leonard JR, Smyth MD et al (2015) Surgical management of complex multiloculated hydrocephalus in infants and children. Childs Nerv Syst 31:243-249

2. Rhoton AL Jr, Gomez MR (1972) Conversion of multilocular hydrocephalus to unilocular. Case report. J Neurosurg 36:348-350

3. Zuccaro G, Ramos JG (2011) Multiloculated hydrocephalus. Childs Nerv Syst 27:1609-1619

4. Balmer B, Bernays RL, Kollias SS, Yonekawa Y (2002) Interventional MR-guided neuroendoscopy: a new therapeutic option for children. J Pediatr Surg 37:668-672

5. Kollias SS, Bernays RL (2001) Interactive magnetic resonance imaging-guided management of intracranial cystic lesions by using an open magnetic resonance imaging system. J Neurosurg 95:15-23

6. Paraskevopoulos D, Biyani N, Constantini S, Beni-Adani L (2011) Combined intraoperative magnetic resonance imaging and navigated neuroendoscopy in children with multicompartmental hydrocephalus and complex cysts: a feasibility study. J Neurosurg Pediatr 8:279-288 